\title{
Business Communication Digitalized: Accelerated Transformations under the New Normal
}

\section{Hyejung Chang}

Editor of Business Communication Research and Practice, Kyung Hee University, Seoul, Korea

Two years have passed since we first heard of the coronavirus, and our work and personal lives are now a far cry from what they once were. Companies have adapted to a "new normal," one that acknowledges that the new virus is here to stay. This new context has forced them to turn to non-face-to-face strategies for customer marketing, internal and external communication, and the overall business process (Uzialko, 2021). These new work environments are accelerating digital transformation, expanding the role of information and communication technology (ICT) plays in companies of all fields (Blackburn, LaBerge, O’Toole, \& Schneider, 2020).

The move toward digitalization in business communication and the general work environment is not a recent phenomenon. Years before the pandemic, the growing reach of ICT and big data analyses had been receiving attention in both academic and mainstream venues. Topics of discussion included chatbot communication with customers and the expanding scope of automation via robotic process automation (RPA), which not only replaces simple formal tasks but also handles unstructured tasks using coworking systems between employees (Adamopoulou \& Moussiades, 2020; Heo \& Lee, 2018).

In this era of social distancing, communication with customers is more important than ever. With this growth of commu-

Received: Jan 5, 2022 Revised: Jan 18, 2022 Accepted: Jan 20, 2022 Corresponding author: Hyejung Chang

School of Management, Kyung Hee University, 26 Kyungheedae-ro, Dongdaemun-gu, Seoul 02447, Korea

Tel: +82-2-961-9432, E-mail: hjchang@khu.ac.kr

This is an Open Access article distributed under the terms of the Creative Commons Attribution Non-Commercial License (http://creativecommons.org/licenses/ by-nc/4.0/) which permits unrestricted non-commercial use, distribution, and reproduction in any medium, provided the original work is properly cited.

Copyright (๑) 2022 Korean Association for Business Communication. nication, however, there has been a growing need to integrate messages from various channels, such as chatting, notifications, phone calls, e-mails, and short message services (SMS). The pandemic has also caused a rise in the use of smart work systems as a growing number of companies have been allowing their employees to work remotely (International Labour Organization, 2020). Business coworking systems now offer messaging, scheduling, project status sharing, file sharing, and communication in one place independent of where employees may be in the world. Many major companies in fields such as finance, manufacturing, law, and health care are introducing business coworking systems into their workforce, and the numbers of companies following suit only seem to be growing.

Many companies have begun to introduce software robots in the process of automating segments of their business to allow their employees to focus on more valuable tasks rather than spend their time on repetitive ones. Software "bots" can work 24 hours a day, 365 days a year. Their productivity is twice as high as that of people, as they can work five times longer than an average person, thus supplying about 10 times the working labor. Once artificial intelligence (AI) is incorporated into these bots, their efficiency will increase by 25 times. These changes are expected to bring dramatic improvements to human productivity while addressing the problems of aging populations and labor shortages. If traditional RPA innovated blue-collar sectors by taking over repetitive tasks, RPAI (RPA + AI) has the potential to innovate white-collar ones, allowing humanity to discover new areas of intelligence (Shin, 2019).

BCRP has thus published a special issue that includes case studies of ICT companies in an effort to better understand how they are using innovative platform strategies in response to the "new normal." These four companies - Uracle, which enhances 
communication with customers through message integration; Madras Check, which provides coworking systems that innovatively change work communication methods; LG CNS, which transforms the corporate environment through optical character recognition (OCR) using AI; and IBM, which seeks hyperautomation to secure work efficiency - are upgrading and innovating message integration, coworking systems, and business process automation.

\section{Integrating Customer Communication Services}

The exponential growth of notifications and SMS messages has recently led to customer fatigue. For many companies, message channels are unstructured, and the rising frequency of messages only leads to higher costs and inefficiency problems. In an effort to overcome these problems, Uracle developed Morpheus, a system that efficiently integrates message channels. Uracle provides a message system for pre-orders until the stage of producing messages, sending them through various channels, collecting results, and providing analysis data (Jung, Kim, \& Shin, 2022).

Morpheus is said to prioritize cost reduction, efficiency maximization, effective communication, and customer satisfaction. In terms of cost reduction, Morpheus uses a sequential sending system that sends messages centered on push, which is a free messaging service, and then sends a message through it as well as an SMS in the order of costs. Additionally, in terms of efficiency, i.e., the sending performance of messages, Morpheus has a $99.9 \%$ success rate through alternative sending functions. A reliable communication tool should allow users to confirm whether the message has been sent, received, and read. Even if the user deletes the app, it can be expanded to a communication tool with potential customers to re-attract customers. It limits the speed and quantity of message sending in response to customer fatigue, especially in situations in which there is potential to be a flood of users, such as in cases of promotions. In such a case, the system can be adjusted to avoid becoming overloaded.

\section{Changes using Coworking Tools}

Implementing a user-centered optimized work environment with digital technology is also a problem that affects corporate productivity. Coworking systems can be a major success point for work innovation as hybrid work environments, which are mixture of face-to-face and non-face-to-face channels, are becoming more commonplace. Madras Check, which is introduced in this paper, has become the leading company in the Korean market by creating their first-generation business co- working system, Flow (Lee \& Cho, 2022).

Flow is an easy-to-use messenger-based system that enables flexible and social collaboration for projects at every level. It is considered a smart work system because it combines history management and systematic workflow functions. Flow is also designed to strengthen partnerships. It allows a project leader to be designated so that anyone can act as the leader regardless of their affiliation or position, and it places no limit on the number of partners that can be invited. Many manufacturers, financial companies, and media companies in Korea are thus satisfied users of Flow who are happy to see improvement in their user work satisfaction in terms of team collaboration and personal work management.

\section{Al-Powered OCR in Robotic Process Automation}

OCR is technology that digitalizes characters acquired through scanning or photographs to allow computers to recognize those characters. It has been widely used in work environments to secure data from scans or image documents, which requires a great deal of manual work. OCR is undergoing a quiet revolution as AI is being integrated with existing OCRs, which was limited in recognition rates. As documents become more diverse and $\mathrm{AI}$ accuracy improves, OCR is becoming essential for the introduction of RPA. In other words, AI-OCR is currently increasing its recognition rate as its accuracy increases with the application of AI algorithms in tasks such as document classification and detection and recognition. Therefore, even in areas related to tasks that cannot be completely automated, corporate interest in the application of next-generation RPA linked to AIOCR has been growing.

As LG CNS' AI-OCR is designed to allow RPA to automatically collect and classify files, check the last steps, and even re-learn misrecognized data, it can reduce errors (Lee, Lim, \& Chung, 2022). In fact, the LG Group has used AI-OCR for quality control and assurance reports, additional costs for overseas transportation, and self-measurement records of air pollution emissions. Additionally, LG Chemical has digitalized all its facilities and has inputted drawing/facility technology data managed offline into the system through AI-RPA.

\section{Next Robotic Process Automation}

The most frequently mentioned word in the field of business automation is "hyperautomation." Hyperautomation refers to a more advanced automation strategy that applies various technologies such as AI and machine learning beyond automation 
in general, increasing work efficiency and improving quality. An important foundation for hyperautomation is RPA solutions.

IBM also provides RPA solutions to automate all types of tasks to realize hyperautomation. IBM's business automation platform provides solutions by dividing the working process of companies into five stages: Discovery, Modeling, Building, Using, and Management areas. In particular, in the building area, IBM's RPA solutions are included, allowing robots to be built through RPA studios with built-in AI instructions. As of 2021, IBM Korea is working on 11 RPA projects, saving companies a total of more than 10,000 working hours (Bu, Jeong, \& Koh, 2022). IBM is thus focusing its efforts on helping companies integrate AI into automation to create insightful information, provide faster service, improve the user experience, and increase profits through digital transformations based on AI.

Many things have changed due as a result of the pandemic, but the biggest change has been the acceleration of digital transformation in the way we communicate and work. As the ICT infrastructure market was already in place even before the pandemic, this situation will not change significantly even after the end of it. Rather, over time, integrated messages, coworking systems, and RPA are likely to be considered key factors for accelerating digital innovation. It is now time for companies to prepare for a post-pandemic work environment. Middle-aged people have become digital users, and students, who are potential members of the company's organization, are now more familiar with the digital environment due to their online classes. With a hybrid work environment that combines online and offline communication, the ICT-based infrastructure market for work process innovation will continue to expand and develop as we get used to non-face-to-face smart work systems. Innovations in business communication patterns will naturally follow.

\section{References}

Adamopoulou, E., \& Moussiades, L. (2020). Chatbots: History, technology, and applications. Machine Learning with Applications, 2(15), 100006.

Blackburn, S., LaBerge, L., O’Toole, C., \& Schneider, J. (2020, April 22). Digital strategy in a time of crisis. McKinsey Digital. Retrieved from https://www.mckinsey.com/business-functions/mckinseydigital/our-insights/digital-strategy-in-a-time-of-crisis

Bu, S., Jeong, U. A., \& Koh, J. (2022). Robotic process automation: A new enabler for digital transformation and operational excellence. Business Communication Research and Practice, 5(1), 2935.

Heo, M., \& Lee, K. J. (2018). Chatbot as a new business communication tool: The case of Naver TalkTalk. Business Communication
Research and Practice, 1(1), 41-45.

International Labour Organization [ILO]. (2020). Teleworking during the COVID-19 pandemic and beyond: A practical guide. Geneva, Switzerland: ILO.

Jung, W., Kim B., \& Shin S. (2022). The use of unified message system as a vehicle for effective mobile business communication: The case of morpheus message. Business Communication Research and Practice, 5(1), 14-19.

Lee, H., \& Cho, H. Y. (2022). Digital transformation for efficient communication in the workplace: Analyzing the flow coworking tool. Business Communication Research and Practice, 5(1), 20-28.

Lee, J. H., Lim, E., \& Chung, N. (2022). LG CNS digital transformation using robotic process automation. Business Communication Research and Practice, 5(1), 36-41.

Shin, C. O. (2019, June 13). Masayoshi son. AI destroys jobs? Creative jobs. Maeil Business Newspaper. Retrieved from https:// www.mk.co.kr/news/business/view/2019/06/416331/

Uzialko, A. (2021, November 11). Workplace automation is everywhere, and it's not just about robots. Business News Daily. Retrieved from https://www.businessnewsdaily.com/9835automation-tech-workforce.html 